\title{
56
}

\section{Environment For Three Dimensional Graphics User Interface Development}

\author{
Y. K. Chan, Sam Kwong, C. H. Lee, and K. L. Chan \\ Department of Computer Science, City Polytechnic of Hong Kong, 83 Tat Chee Avenue, Kowloon \\ Tong, Hong Kong
}

\begin{abstract}
Graphics User Interface(GUI) serves as a link between the end user of an application and the application itself. Run time GUI is characterized by its look-and-feel and interaction style. Characteristics of a run time GUI system depends on the particular GUI building tool used and its underlying architecture. Current implementation of GUI are in two-dimensions(2D) and the trend is to extend GUI into three-dimensions(3D) in order to provide a natural interface for applications in 3D. It is expected that 3D specific GUI builder will become available when methodology for automating 3D GUI building process for a large class of 3D application is better understood. Until then, it is proposed that a 3D development and debugging environment should be available for building such interfaces for individual application as required. This paper describes the overall framework and some details that enables the building of 3D GUI.
\end{abstract}

Keycodes: D.1.7; D.2.5; I.3.2

Keywords: Visual Programming Language, Problem Analysis Diagram, Computer Aided Diagram, Visual Programming Environment, Graphics Kernal System, XLIB, Graphics User Interface

\section{Introduction}

Progress in the area of visual languages[1,2] for different applications have been reported and in turn motivated a visual programming environment(VPE)[3] that is specially architected to support the development and debugging of graphics applications.

The VPE can be considered as a lower CASE tool and is therefore suitable in developing applications [4] in which the software architecture is already specified. The VPE can also be applied to implement the Xlib part of the X Window System which is a network-transparent window based graphics system. As the Xt Intrinsic defines an architectural model for widget, GUI run time system such as Motif[5] can also be implemented by the VPE. It should be noted that although the Motif widget set is 3D in appearance, the contents of icon objects are not truly 3D. The need for rapid prototyping of GUI is reflected both in the area of research[6] and in the area of products[7]. However, existing User Interface methodology and tools can not adequately mect User Interface requirement of a 3D nature[8]. Until rapid prototyping for 3D GUI becomes mature, it is envisaged that complex 3D User Interface architecture will have to be derived through specification obtained by formal methods. The subsequent specification that yields the corresponding architecture can then be implemented in VPE and the appropriate use of functions in GKS-3D[9].

2. The VPE: the visual programming language(VPL) 
The VPE is a visual programming environment that separates ' $C$ ' source code procedure into three parts. These three parts are the "Data Declaration and Initialization" part, the "Control of Flow using Problem Analysis Diagram(PAD)[10]" part and the "Executable Assignment Statements" part, respectively. A subset of the VPL syntax is presented as an extended BNF notation in Appendix 1.

The three parts in the VPL are illustrated in Figures 1, 2 and 3. Figure 1 and 2 illustrate how flow and execution are presented in VPL. It can also be deduced that, from Figures 1 and 2, icons can be inserted and deleted at will to alter the control flow and that there are certain viewing flexibility in the VPL system. Figure 3 is self explanatory by virtue of its contents for data declaration and initialization.

It is advantages to use VPL instead of representing a program procedure in terms of a piece of flat text when referred to the dimensional analysis proposed by Shu[11]. The visual extent[11] of VPL over a piece of ' $C$ ' source code increases the readability and maintainability of the target software. The VPL enforces programmer discipline by imposing structure at the program development stage.

\section{The VPE: the debugger for graphics applications}

Figures 4, 5, 6 and 7 are four snap shots during the debugging of a simple graphics application. The upper right window is the debugger and the lower left window is the debuggee. It should be noted that the two windows should really have been initiated to larger sizes to cover more of the display surface of the graphics workstation display monitor. The default 'trace' level during debugging is at icon trace level which means that a 'step' command advances execution by one icon. In Figure 5, the lines of code for 'graphics initialization for debuggee', 'clear graphics and text contents in debuggee', 'initialize locator input class with mouse as a physical input device', 'initialize $x=y=0$ ' has NOT yet been executed. These four statements will be executed if user types in either 'step<cr>' or simply '<cr>' from keyboard. The visual appearance of Figure 7 indicates that the 'locator position has been defined' and that 'the line positioned between $x=y=0$ and locator'. It should be noted that the code in Figure 6 would not have been executed if the cross-hair cursor position is not moved to the debuggee window. The sharing of input resources between debugger and debuggee and that the debuggee execution may either be suspended or nunable based on input context has also been reported[12].

Other debugging features include variable trace, execution until breakpoint, etc..

\section{Similarity between GKS-2D functionality and Interactive Objects[13]}

The Interactive Objects(IO) as proposed by Wood and Gray have three common attributes(visibility, selectability and movability). In the context of GKS-2D, the contents of an Object are OUTPUT PRIMITIVES and the Object itself is a NAMED SEGMENT containing all the output primitives. Visibility and selectability as defined in [13] can then be implemented by GKS-2D segment attribute such as invisible/visible, undetectable/detectable. The movability attribute can be implemented by SEGMENT transform in which translate- $x$ and translate-y are the magnitudes to define distance moved. Additional settable segment attributes are highlighting and priority. Other SEGMENT manipulation capability through the use of segment transform include segment scaling and rotation.

\section{An example of IO implementation using GKS-2D}

Implementation of IO attributes by GKS-2D functionality is illustrated in Appendix 2. The implementation is in Fortran binding.

Appendix 2a defines the transformation number. All GKS segments(IO) are within this transformation number 10. In the context of GUI, it is useful to have a transformation number for IOs categorized under application and another number for IOs categorized under development tools, etc..

Appendix $2 \mathrm{~b}$ illustrate how the fourth segment can be created. 
Appendix 2c illustrates how a segment can be picked and then subsequently translated if required.

\section{Conclusion}

Infrastructure for developing 2D IO based on VPE and the suitable use of GKS-2D functions have been presented. Extension of VPE capability to cater for 3D graphics development and debugging requires no more than perspective and parallel projection[14] of 3D data. Such additional capability does not require structural change in the VPE and therefore modification to VPE is straight forward to catering for 3D 10 development resulting in an enhanced VPE for 3D application development. The advantage of structural compatibility between 2D run time GUI system and 3D run time GUI system developed using the same methodology is obvious not be overlook in terms of compatibility and maintenance and well as programmer portability.

\section{REFERENCES}

1. Chang S.K., "Visual Languages", Principles of Pictorial Information Systems Design, Prentic-Hall International Editions, 1989, pp262-300.

2. Williams C.S., Rasure J.R., "A Visual Language for Image Processing", IEEE Workshop on Visual Languages, 1990, pp86-91.

3. Tam S.S. and Chan Y.K., "Graphics Application Development under Window Environment", Proc. of 2nd Int. Conf. in Software Eng. \& Knowledge Eng., Illinois, U.S.A., June 1990, pp234-238.

4. International Organisation for Standardization: Information Processing Systems - Computer Graphics - Graphics Kernel System(GKS) - Functional Description. ISO 7942(1985).

5. Open Software Foundation, OSF/Motif Style Guide, 1990.

6. Philip G. Gray, Alistair C. Kilgour and Catherine A. Wood, "Dynamic reconfigurability for fast prototyping of user interfaces", Software Engineering Journal, November 1988, pp257-262.

7. Asymetrix Corporation, "Using Toolbook, a Guide to Building and Working with Books", Bellevue, WA. 1989.

8. A. D. Bimbo, M. Campanai and P. Nesi, "A Three-Dimensional Iconic Environment for Image Database Querying", IEEE Trans. on Software Engineering, vol. 19, no. 10. Oct 1993, pp9971011.

9. International Organisation for Standardization: Information Processing Systems - Computer Graphics - Graphics Kernel System for Three Dimension(GKS-3D) - Functional Description. ISO/DIS 8805(1986).

10. Futamura Y., Kawai T., Horikoshi H. and Tsutsumi M., "Development of Computer Program by PAD(Problem Analysis Diagram)", Proceedings of the 5th International Software Engineering Conference, 1981, pp.325-332.

11. Shu Nan C., "Visual Programming Languages: A Perspective \& A Dimensional Analysis", Visual Languages edited by S. K. Chang et al., Plenum Press, New York, 1986.

12. Elliot A. and Sachs G.M., "Shering Input Devices in the Starbase/X11 Merge System", HewlettPackard Journal, Dec., 1989, pp.38-41

13. Wood C.A. and Gray P.D., "User Interface - Application Communication in the Chimera User Interface Management System", SOFTWARE Practice \& Experience, Vol. 22, No. 1, Jan. 1992. pp. 63-84.

14. Rogers D.F. and Adams J.A.,"Mathematical Elements for Computer Graphics", McGraw Hill, 1976. ISBN 0-07-053527-2. 


\section{Appendix I}

A subset of the VPL syntax

The followings are the extended BNF representation for the meta-language of the visual language used on the PAD diagram.

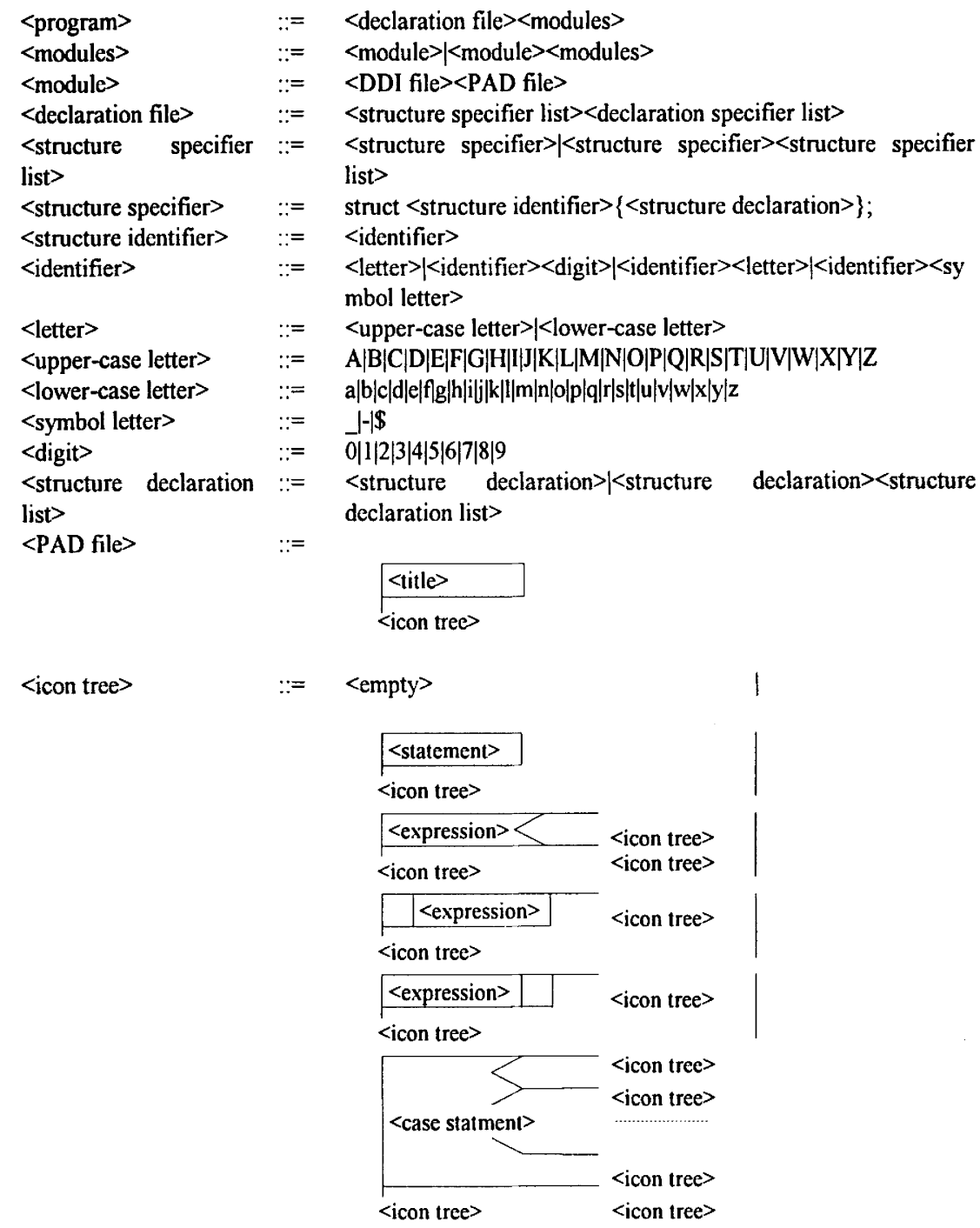

$<$ structure declaration $>\quad:=\quad<$ type specifier $><$ declaration $>$; 
Appendix II

a)

c all segments defined inside transformation number 10

c TRANSFORMATION number 10

c by setting window, setting viewport, select transformation number

CALL GSWN $(10,0.0,10.0,0.0,10.0)$

CALL GSVP $(10,0.0,1.0,0.0,0.72)$

CALL GSELNT ( 10 )

c set input priority for transform 10 greater than transform 0

CALL GSVPIP ( $10,0,0)$

c set workstation windown and workstation viewport

CALL GSWKWN (IWKID, 0.0, 1.0, 0.0, 0.72)

CALL GSWKVP (IWKID, 0.0, 0.27, 0.0, 0.195)

(b)

c now create segment for DUCK

CALL GCRSG(IFOUR)

CALL GFA ( NUMDUCK, DUCKX, DUCKY)

CALL GCLSG

c)

c PICK any one of the six segments

c default Prompt \& Echo Type is Device Coordinate echo plus pick symbol

c mouse, \{none,ok\}, segment, pickid

7700 CALL GRQPK ( IWKID, IONE, ISTATUS, ISEGMENT, IPKID ) IF (ISTATUS .EQ. 0 ) GOTO 7700

c TRANSLATE the segment that has been picked (selected)

c Prompt \& Echo Type 12 is World Coordinate digital value plus cross-hair

CALL GINLC( IWKID, 1, 10, XINITLC(ISEGMENT), YINITLC(ISEGMENT), $12,0.0,0.1,0.0,0.1,0$, NULLSTR80)

CALL GSLCM ( IWKID, $1,1,1$ )

7010 NORTRANS $=10$

CALL GSMLC ( IWKID, 1, NORTRANS, XLC, YLC )

IF ( NORTRANS .NE. 10) GOTO 7010

DXLC $=$ XLC - XINITLC(ISEGMENT)

DYLC $=$ YLC - YINITLC(ISEGMENT)

TM(I,J) = ETM(ISEGMENT,I,J) [ I=1,2; J=1,3 ]

CALL GACTM ( TM, XNNITLC(ISEGMENT),YINITLC(ISEGMENT), DXLC,DYLC, 0.0, 1.0,1.0, 0, TMO )

XINITLC(ISEGMENT) $=$ XLC

YINITLC(ISEGMENT) $=$ YLC

ETM(ISEGMENT,I,J) = TMO(I,J) $[\mathrm{I}=1,2 ; \mathrm{J}=1,3]$

IF ( DXLC.NE.0.0 .OR. DYLC.NE.0.0)CALL GSSGT ( ISEGMENT,TMO)

CALL GESC ( 100, 1, STR80)

IF ( STR80(1:1) .EQ.'2' .OR. STR80(2:2) .EQ. '0') GOTO 7010

CALL GSLCM ( IWKID, 1, 0, 1)

CALL GRSGWK ( IWKID ) 


\section{Appendix III}

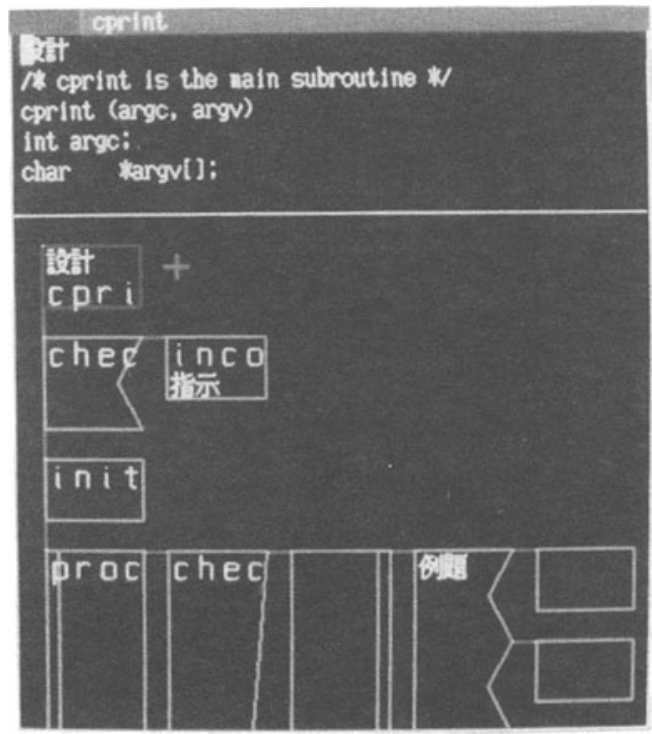

Figure 1. a Problem Analysis Diagram example

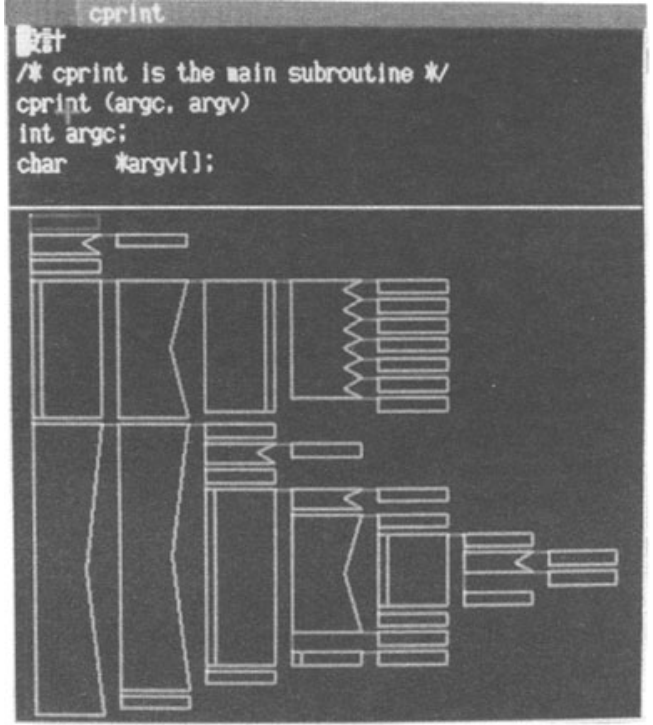

Figure 2. a shrunk Problem Analysis Diagram 


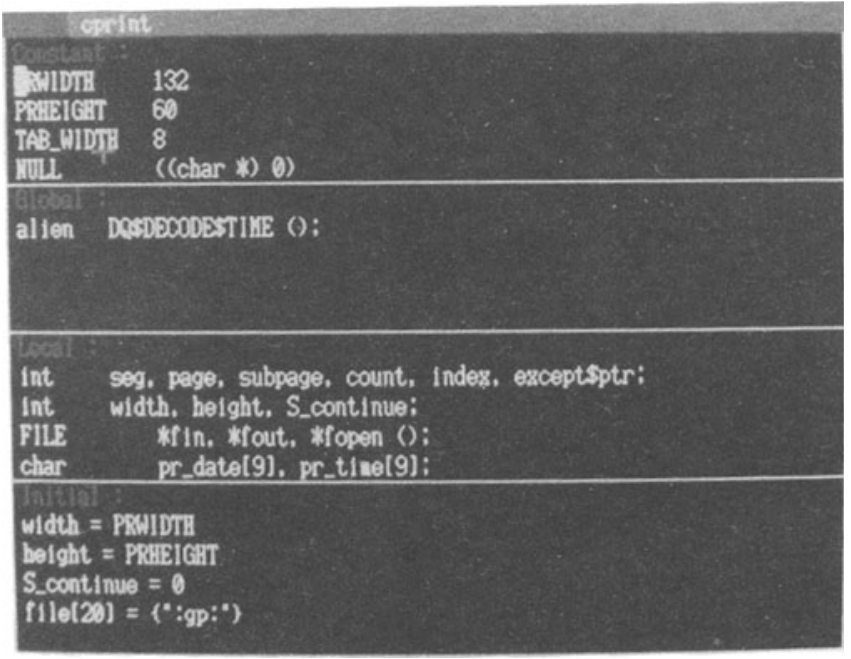

Figure 3. a Data Declaration and Initialization example

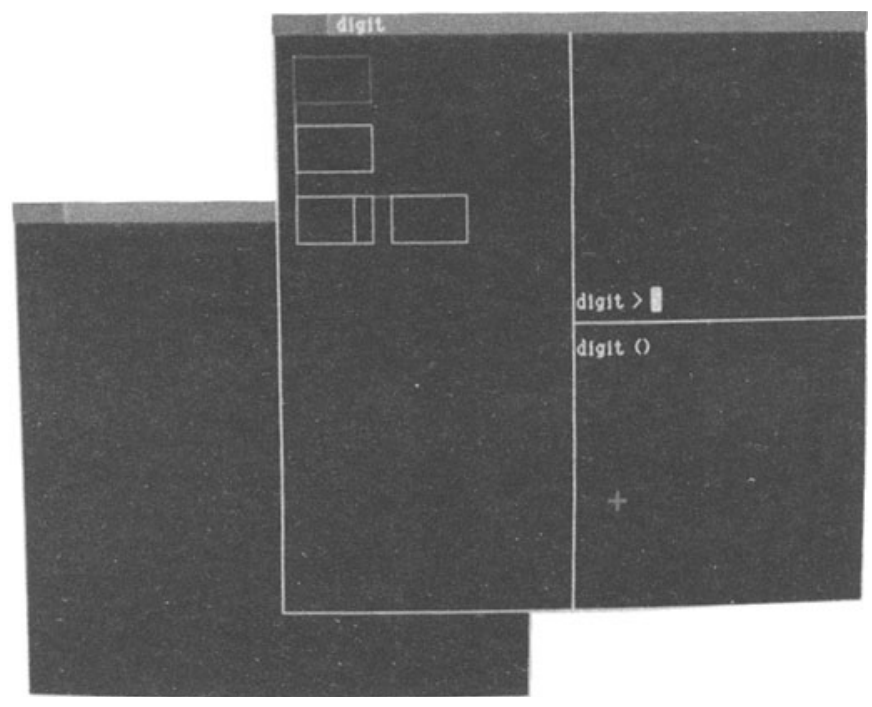

Figure 4. Initializing Debugger and Debuggee 


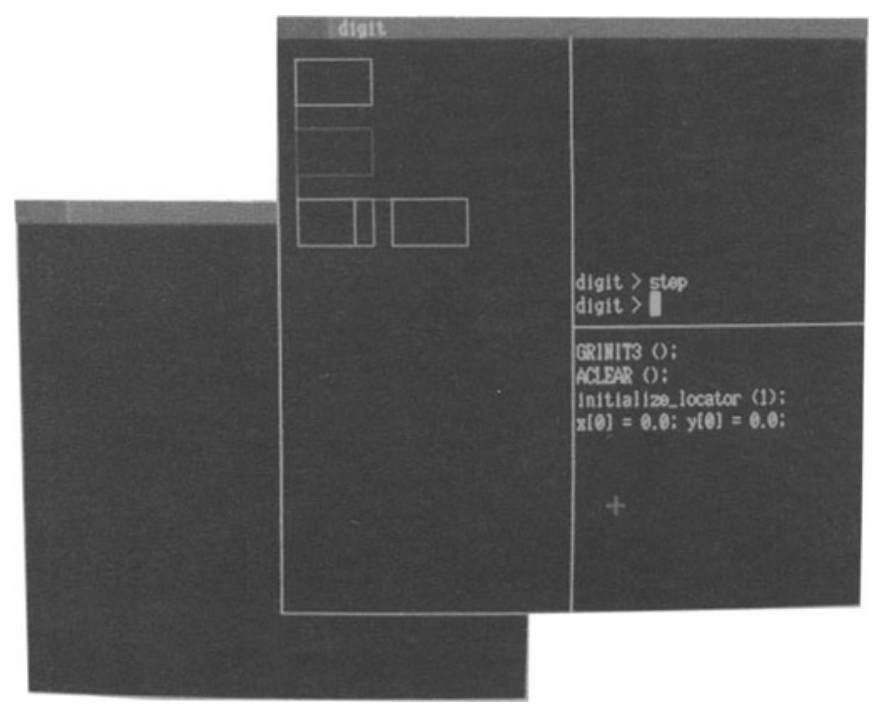

Figure 5. single stepping debugging

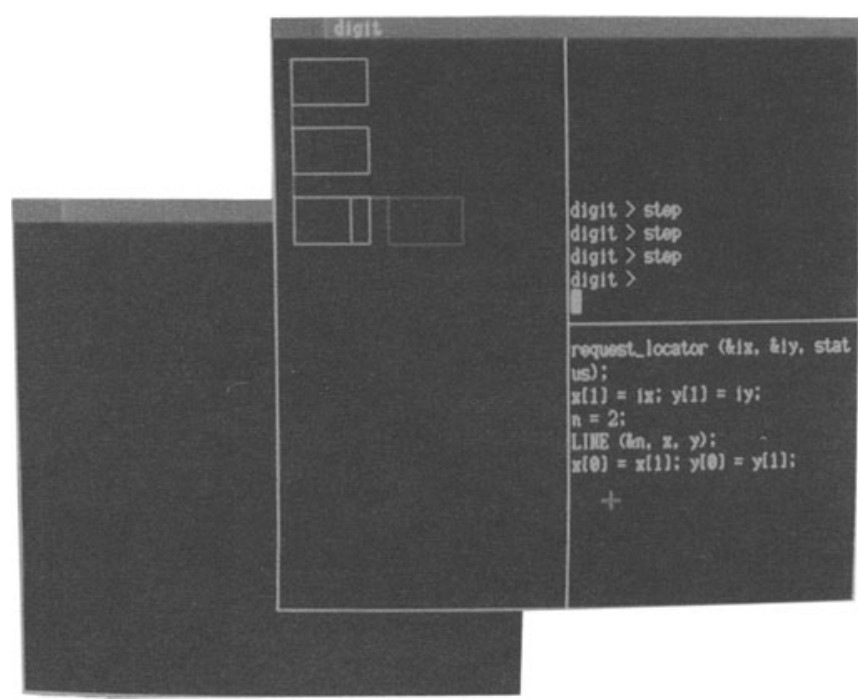

Figure 6. graphics application debugging 


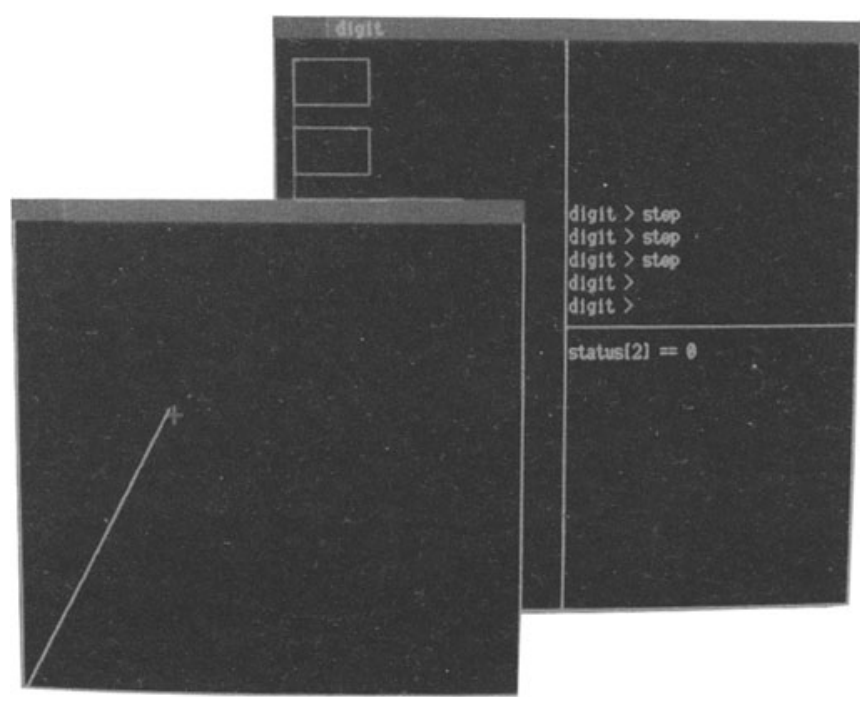

Figure 7. sharing of debugger/debuggee input resource 